\title{
Halo chemistry and first stars. The chemical composition of the matter in the early Galaxy, from $\mathrm{C}$ to $\mathrm{Mg} \dagger$
}

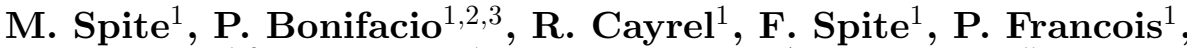
H. G. Ludwig ${ }^{1,2}$, E. Caffau ${ }^{1}$, S. Andrievsky ${ }^{4}$, B. Barbuy ${ }^{5}$, B. Plez ${ }^{6}$,

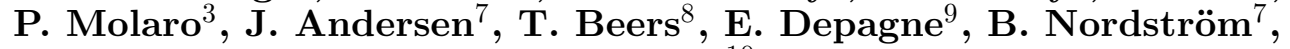
F. Primas ${ }^{10}$

${ }^{1}$ GEPI - Observatoire de Paris-Meudon F92195 Meudon Cedex, France email: monique.spite@obspm.fr

${ }^{2}$ CIFIST Marie Curie Excellence Team

${ }^{3}$ INA - Osservatorio Astronomico di Trieste, Via Tiepolo 11, I-34143 Trieste, Italy

${ }^{4}$ Dept of Astronomy, Odessa Nat. University, Shevchenko Park, 65014 Odessa, Ukraine

${ }^{5}$ Univ. de Sao Paulo, Depto. de Astronomia, Rua do Matao 1226, Sao Paulo 05508-900, Brazil

${ }^{6}$ GRAAL, Univ. de Montpellier II, F-34095 Montpellier Cedex 05, France

${ }^{7}$ The Niels Bohr Institute, Astronomy group, Juliane Maries Vej 30, DK-2100 Copenhagen, Denmark

${ }^{8}$ Michigan State Univ., East Lansing, MI 48824, USA

${ }^{9}$ Las Cumbres Observatory, Goleta, CA 93117, USA

${ }^{10}$ ESO, Karl Scwarzschild-Str. 2, D 85749, Garching bei München, Germany

\begin{abstract}
From NLTE computations of the magnesium abundance in a sample of extremely metal-poor giants we derive $[\mathrm{Mg} / \mathrm{Fe}]=+0.7$, leading to $[\mathrm{Al} / \mathrm{Mg}]=-0.80$ and $[\mathrm{Na} / \mathrm{Mg}]=-0.85$ in the early Galaxy. The ratio $[\mathrm{O} / \mathrm{Mg}]$ should be near to the solar value. Measurements of nitrogen abundances derived from the analysis of the $\mathrm{NH}$ band in eight more stars confirm the large scatter of the ratios $[\mathrm{N} / \mathrm{Fe}]$ and $[\mathrm{N} / \mathrm{O}]$ in the early Galaxy.
\end{abstract}

Keywords. Stars: abundances, stars: atmospheres, stars: Populations II, Galaxy:abundances, Galaxy:evolution, nucleosynthesis

\section{Introduction}

The "Extremely metal-poor" stars formed less than 1 Gyr after the formation of the Galaxy. They are extremely metal-poor because, at that time, the matter had been enriched by a very small number of supernovae. Moreover, these first supernovae could only be massive type II supernovae which have a very short lifetime.

In contrast, the chemical composition in the Galactic disk is the result of enrichment not only by SNII, but also by AGB stars and SNI.

Seven years ago, to take advantage of the high-resolution spectrograph UVES at the VLT, and of the existence of a large sample of EMP stars (H\&K survey: Beers, Preston \& Schectman 1992), Roger Cayrel stimulated the formation of a "First stars team" to apply for an ESO Large Program dedicated to the study of the chemical composition of the Galactic matter in the early times. Other teams in the world have chosen to work on similar subjects, in particular at the Keck and SUBARU telescopes in Hawaii or at

$\dagger$ Based on observations made with the ESO Very Large Telescope at Paranal Observatory, (Large Programme "First Stars", ID 165.N-0276; P.I.: R. Cayrel, and Programme 078.B-0238; P.I.: M. Spite). 
the AAT in Australia. Generally speaking, there is a rather good agreement between the results of these different teams.

\section{Observations and Spectrum analysis}

In summary, 52 very metal-poor stars $(34$ with $[\mathrm{Fe} / \mathrm{H}]<-2.9)$ were observed in the "First Stars" programme $(\mathrm{S} / \mathrm{N} \approx 200, \mathrm{R} \approx 45000,3350<\lambda<10000)$. All these stars were "normal" metal-poor stars, not carbon-enriched, but some carbon-rich stars were observed for comparison. Later in a complementary run focused on the problem of the nitrogen abundance, ten more giant stars in the lower part of the HR diagram were observed in the same conditions.

In a first step, a classical LTE analysis was performed using MARCS theoretical 1D plane-parallel model atmospheres (Gustafsson et al. 2008). The stellar effective temperature was determined from photometry (or the wings of the $\mathrm{H} \alpha$ line, for dwarfs) and the gravity from the ionization equilibrium of iron.

More details can be found in Cayrel et al. (2004) and Bonifacio et al. (2007, 2008).

\section{3. $[\mathrm{O} / \mathrm{Mg}],[\mathrm{Na} / \mathrm{Mg}]$ and $[\mathrm{Al} / \mathrm{Mg}]$ in the Galaxy, Influence of NLTE effects.}

In extremely metal-poor stars, the lines are often very weak and the abundance of several elements like sodium or aluminum can be determined only from the resonance lines. However, it is well known that these lines are very sensitive to NLTE effects. As a first approximation we applied a systematic non-LTE correction actually estimated for dwarfs to all the stars (Cayrel et al. 2004). But in both cases the scatter in the abundance ratios was very large; for $\mathrm{Na}$ there was strong disagreement between dwarfs and giants. After a complete NLTE computation of the $\mathrm{Na}$ and $\mathrm{Al}$ resonance lines, the agreement between the $\mathrm{Na}$ and $\mathrm{Al}$ abundances in giants and in dwarfs became very good and the scatter much smaller (Andrievsky et al. 2007, 2008).

But as noted by Gehren et al. (2006) non-LTE effects are also not negligible when computing $\mathrm{Mg}$ abundances in metal-poor stars. Therefore we decided to compute also NLTE profiles for Mg lines. For giants we found a mean NLTE correction of about +0.4 dex and $[\mathrm{Mg} / \mathrm{Fe}] \approx+0.7$ (computations for dwarfs are in progress). In Cayrel et al. (2004) we measured $[\mathrm{O} / \mathrm{Fe}] \approx+0.7$ (without $3 \mathrm{D}$ correction). This measurement of the oxygen abundance is not sensitive to NLTE effects (forbidden line). The new value of the magnesium abundance in giants would imply $[\mathrm{O} / \mathrm{Mg}] \approx+0.0$ in the early Galaxy.

In figures 1 and 2 we show the variation of the ratios $[\mathrm{Al} / \mathrm{Mg}]$ and $[\mathrm{Na} / \mathrm{Mg}]$ in the Galaxy. For the disk stars $[\mathrm{Al} / \mathrm{Mg}]$ is larger than $-0.1 \mathrm{dex}$ (Gehren, 2006), in the halo this ratio decreases, the minimum is reached at about $[\mathrm{Fe} / \mathrm{H}]=-3.0$ with $[\mathrm{Al} / \mathrm{Mg}]=-0.8$, then at lower metallicity it seems to increase slightly.

At very low metallicity the scatter of $[\mathrm{Na} / \mathrm{Mg}]$ is larger than for $[\mathrm{Al} / \mathrm{Mg}]$, but all the "Na-rich" stars are "mixed" stars. These stars have a high N abundance coupled with a low $\mathrm{C}$ abundance and a low value of the ${ }^{12} \mathrm{C} /{ }^{13} \mathrm{C}$, ratio, indicating a mixing with the $\mathrm{H}$ burning layer. If this mixing is particularly deep, it can bring products of the $\mathrm{O}, \mathrm{Ne}, \mathrm{Na}$ cycle to the surface (Denissenkov \& Pinsonneault 2008). The relation between $[\mathrm{Na} / \mathrm{Mg}]$ and $[\mathrm{Fe} / \mathrm{H}]$ is a continuous enrichment through all three galactic populations, halo, thick disk, thin disk. The minimum is reached at about $[\mathrm{Fe} / \mathrm{H}]=-3.0$ with $[\mathrm{Na} / \mathrm{Mg}] \approx-0.85$, then at lower metallicity it is possible that $[\mathrm{Na} / \mathrm{Mg}]$ increases slightly. 


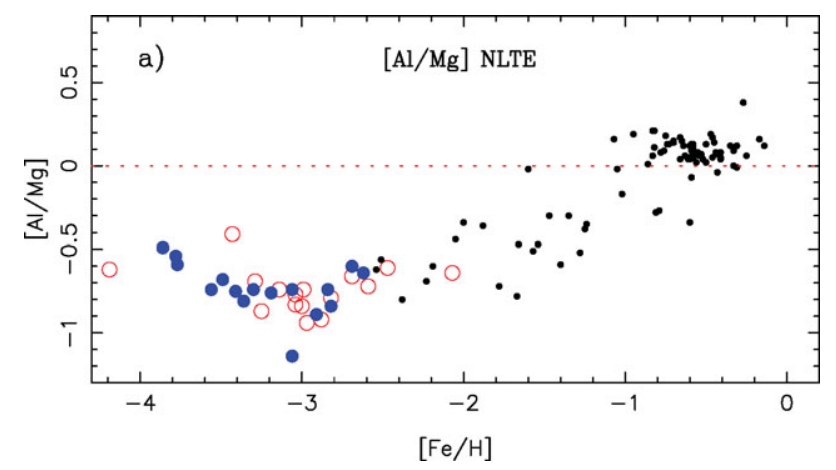

Figure 1. Evolution of the $[\mathrm{Al} / \mathrm{Mg}]$ ratio in the Galaxy as a function of $[\mathrm{Fe} / \mathrm{H}]$. The small black dots are the data by Gehren et al. $(2004,2006)$ for the disk and the halo. The large blue dots represent our sample of "unmixed" giants, the open circles the "mixed" giants.

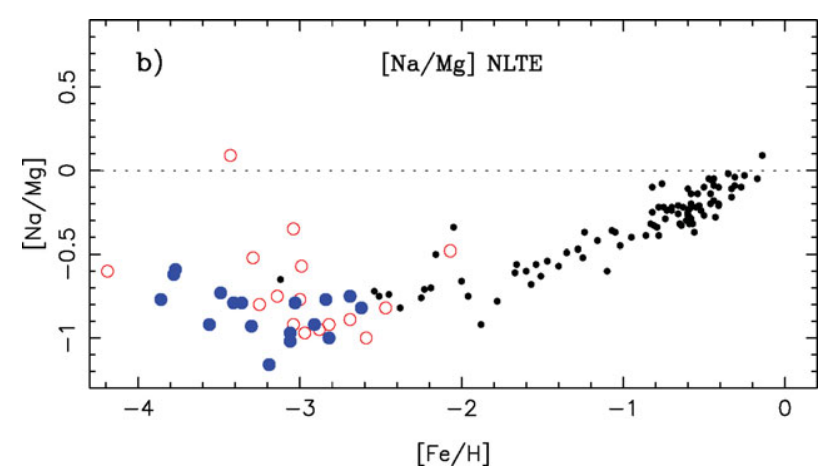

Figure 2. Same as Fig. 1, but for $[\mathrm{Na} / \mathrm{Fe}]$. At very low metallicity the scatter is larger than for $[\mathrm{Al} / \mathrm{Mg}]$ but all the "Na-rich" stars are mixed stars, and the enrichment is probably due to mixing with the deep $\mathrm{H}$ burning layer.

\section{4. $\mathrm{C}$ and $\mathrm{N}$ abundances in the early Galaxy}

In extremely metal-poor stars, carbon and nitrogen abundances are derived from the $\mathrm{G}$ band of $\mathrm{CH}(430 \mathrm{~nm})$ and the violet band of $\mathrm{NH}(336 \mathrm{~nm})$. In general, the $\mathrm{NH}$ (and $\mathrm{CN}$ ) bands are not visible in the spectra of EMP turnoff stars (the stars are too hot), so the $\mathrm{N}$ abundance can be determined only in giants. On the other hand, the abundances of $\mathrm{C}$ and $\mathrm{N}$ in the pristine gas can be deduced only from stars located below the bump in the HR diagram. At higher luminosity, the atmospheres of the stars are significantly altered by mixing and thus are not a good diagnostic of the initial chemical composition (Spite et al., 2005, 2006).

In Spite et al. (2005), we showed from our sample of unmixed stars that the N abundance in the early Galaxy showed large scatter. But since there were only few unmixed giants, we could not decide between a simple scatter, two different levels of abundance, or even an anti-correlation between $[\mathrm{Fe} / \mathrm{H}]$ and the $[\mathrm{N} / \mathrm{Fe}]$ or $[\mathrm{N} / \mathrm{Mg}]$ ratios.

We have recently observed the region of the $\mathrm{NH}$ band in eight more "unmixed" EMP giants, selected from the sample of Barklem et al. (2005). The N abundances in these stars have been computed from synthetic spectra. The results are shown in Fig. 3. These new measurements support the existence of significant scatter in $[\mathrm{N} / \mathrm{Fe}]$ in EMP stars. This scatter could reflect different rotational velocities in the massive first stars (e.g. Meynet et al., 2008). The scatter seems to be even smaller at very lowest metallicities. 


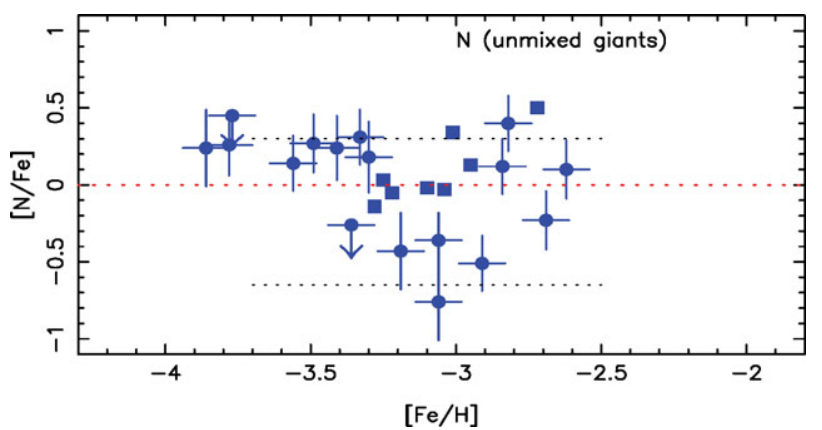

Figure 3. $[\mathrm{N} / \mathrm{Fe}]$ in EMP giants. Our new measurements are shown as squares.

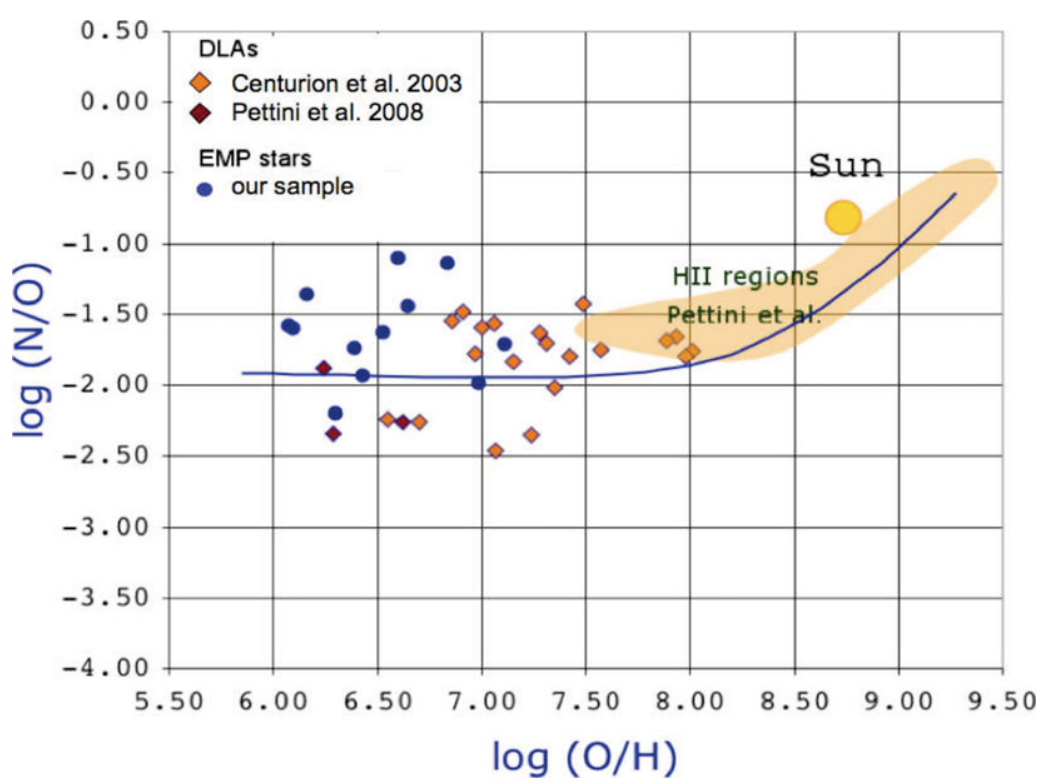

Figure 4. $\log \mathrm{N} / \mathrm{O}$ for $\mathrm{H} 2$ regions, DLAs (diamonds) and EMP stars (blue dots)

However, for $[\mathrm{Fe} / \mathrm{H}]<-3.4$ the $\mathrm{NH}$ band is only measurable if $[\mathrm{N} / \mathrm{Fe}]>0$, so the smaller scatter in this domain could be a spurious effect.

In three of the newly observed stars it was possible to measure the oxygen abundance from the forbidden O I line (see Cayrel et al. 2004). These measurements are in good agreement with the previous measures.

For all the EMP stars with measured $\mathrm{N}$ and $\mathrm{O}$ abundances, the ratio $\mathrm{N} / \mathrm{O}$ can be compared to the values obtained in DLAs by Centurion et al. 2003, or Pettini et al. 2008 (Fig. 4). At low metallicity the scatter of the ratio $\mathrm{N} / \mathrm{O}$ is large in both stars and DLAs. There is no indication of a decrease of $\mathrm{N} / \mathrm{O}$ with $\mathrm{O} / \mathrm{H}$ as it would be expected if the formation of $\mathrm{N}$ would depend on the metallicity of the progenitor. In fact, the mean $\mathrm{N} / \mathrm{O}$ ratio remains almost constant. Massive stars may contribute significantly to the nucleosynthesis of $\mathrm{O}$, but also of $\mathrm{C}$ and $\mathrm{N}$ (Meynet et al., 2008). 


\section{Conclusions}

Precise computations of the abundances of the light metals $\mathrm{Na}, \mathrm{Al}$ and $\mathrm{Mg}$ in extremely metal-poor stars have shown that it is important to take NLTE effects into account. From our preliminary NLTE computations of the Mg abundance in EMP giant stars, we found that: $[\mathrm{Mg} / \mathrm{Fe}] \approx+0.7 \mathrm{dex},[\mathrm{Al} / \mathrm{Mg}] \approx-0.80 \operatorname{dex}$ and $[\mathrm{Na} / \mathrm{Mg}] \approx-0.85 \operatorname{dex}$.

We note that all the ratios $[\mathrm{X} / \mathrm{Mg}]$ in Cayrel et al. (2004) would have to be corrected in the same way, and in particular, the mean value of $[\mathrm{O} / \mathrm{Mg}]$ at very low metallicity could be close to the solar value.

New observations of a sample of "unmixed" metal-poor giants confirm the large scatter of the ratios $\mathrm{N} / \mathrm{O}$ and $\mathrm{N} / \mathrm{Fe}$. The mean value of $\mathrm{N} / \mathrm{O}$ is almost constant in the interval $6<\log (\mathrm{O} / \mathrm{H})<7.5$.

\section{References}

Andrievsky, S. M., Spite M., Korotin, S. A., Spite, F., Bonifacio P., Cayrel, R., Hill, V., \& François, P., 2007, A\&\&A 464, 1081

Andrievsky, S. M., Spite, M., Korotin, S. A., Spite, F., Bonifacio, P., Cayrel, R., Hill, V., \& François, P., 2008, A\&A 481, 481

Barklem, P. S., Christlieb, N., Beers, T. C., Hill, V., Bessell, M. S., Holmberg, J., Marsteller, B., Rossi, S., Zickgraf2, F.-J., \& Reimers, D., 2005, A\&A 439, 129

Beers, T. C., Preston, G. W., \& Schectman, S. A., 1992, AJ 103, 1987

Bonifacio, P., Molaro, P., Sivarani, T., Cayrel, R., Spite, M., Spite, F., Plez, B., Andersen, J., Barbuy, B., Beers, T. C., Depagne, E., Hill, V., François, P., Nordström, B., \& Primas, F., 2007, A $B A$ 462, 851, ("First Stars VII")

Bonifacio, P., Spite, M., Cayrel, R., Hill, V., Spite, F., François, P., Plez, B., Ludwig, H-G., Caffau, E., Molaro, P., Depagne, E., Andersen, J., Barbuy, B., Beers, T. C., Nordström, B., \& Primas, F., 2004, A $\dot{E} A$ submitted, ("First Stars XII")

Cayrel, R., Depagne, E., Spite, M., Hill, V., Spite, F., François, P., Plez, B., Beers, T. C., Primas, F., Andersen, J., Barbuy, B., Bonifacio, P., Molaro, P., \& Nordström, B., 2004, A $\& A 416$, 1117, ("First Stars V")

Centurion, M., Molaro, P., Vladilo, G., Péroux, C., Levshakov, S. A., \& D' Odorico, V., 2003, $A \& 3 A 403,55$

Denissenkov, P. A. \& Pinsonneault, M., 2008, ApJ 679, 1541

Gehren, T., Liang, Y. C., Shi, J. R., Zhang, H. W., \& Zhao, G., 2004, A\&\&A 413, 1045

Gehren, T., Shi, J. R., Zhang, H. W., Zhao, G., \& Korn, A. J., 2006, A\&6A 451, 1065

Gustafsson, B., Edvardsson, B., Eriksson, K., Jørgensen, U. G., Nordlund, A., \& Plez, B., 2008, $\operatorname{arXiv0805.0554v1}$

Meynet, G., Ekstrom, S., Georgy, C., Maeder, A., \& Hirschi, R., 2008, arXiv0806.4063M

Pettini, M., Zych, B. J., Steidel, C. C., \& Chaffee, F. H. 2008 Mont. Not. R., Astron. Soc. 385, 2011

Spite, M., Cayrel, R., Plez, B., Hill, V., Spite, F., \& Depagne, E., François, P. Bonifacio, P. Barbuy, B. Beers, T. Andersen, J. Molaro, P., Nordström, B., Primas, F., 2005, A $\& A 430$, 655, ("First Stars VI")

Spite, M., Cayrel, R., Hill, V., Spite, F., François, P., Plez, B., Bonifacio, P., Molaro, P., Depagne, E., Andersen, J., Barbuy, B., Beers, T. C., Nordström, B., \& Primas, F., 2006, A 6 A 455, 291, ("First Stars IX") 


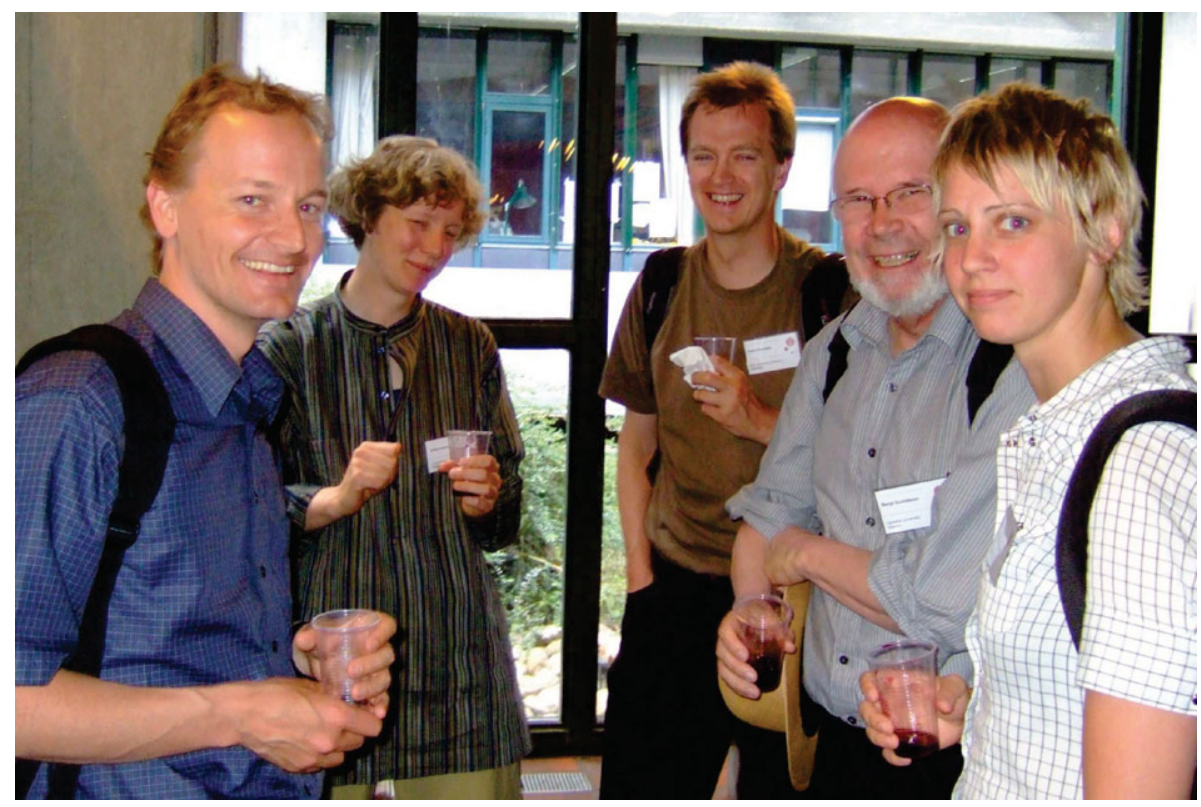

A stellar abundance gang relaxing outside the lecture hall: Andreas Korn, Sofia Feltzing, Frank Grundahl, Bengt Gustafsson, and Anna Önehag.

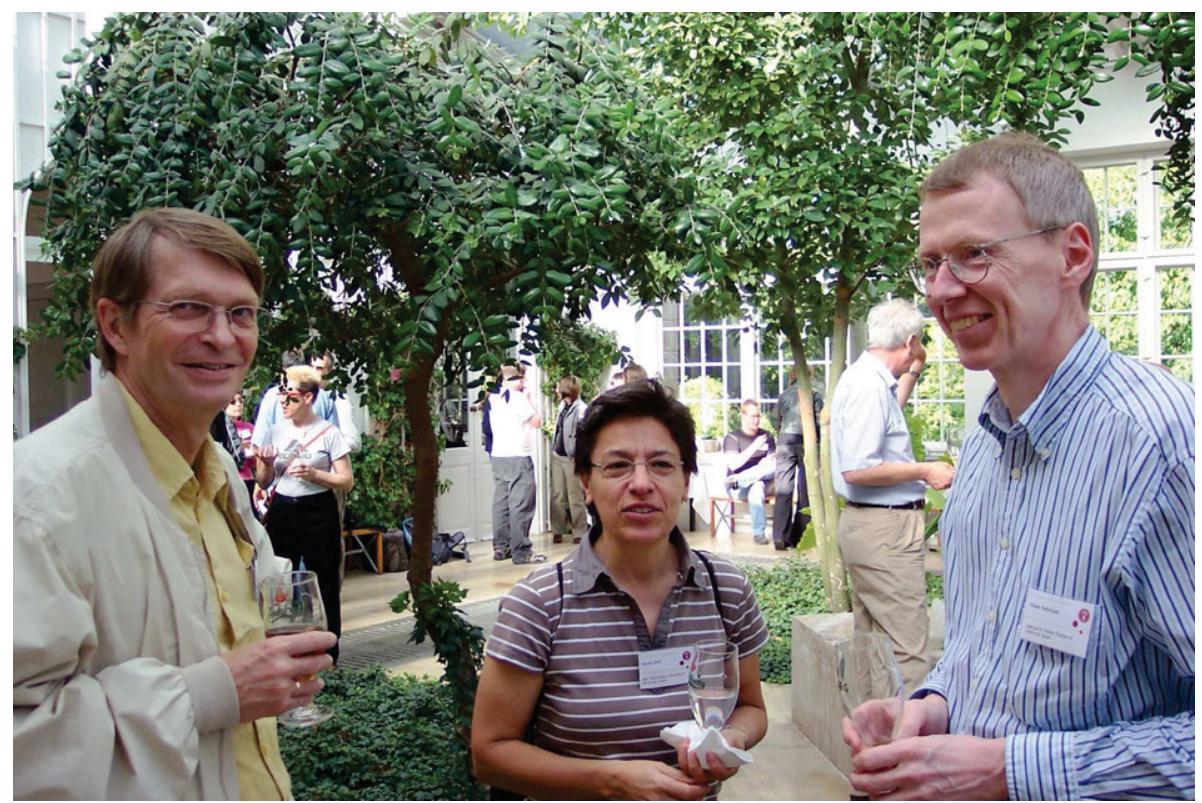

Preben Grosbøl, Carme Jordi, and Claus Fabricius at Carlsberg. 